\section{Genome Sequence Resource of the Causal Agent of Persimmon Anthracnose, Colletotrichum horii Strain SD010 from China}

\author{
Jie Wang, Xianmei Yu, Chengxiang $\mathrm{Ai}^{\dagger}{ }^{\dagger}$ and Rui Gao ${ }^{\dagger}$ \\ Shandong Institute of Pomology, Taian, 271000, China
}

\begin{abstract}
Colletotrichum horii is a main causal agent of persimmon (Diospyros kaki) anthracnose and is distributed widely in persimmon-producing areas of the world. Here, we report the first high-quality draft genome sequence of $C$. horii strain SD010. This will provide a reference for understanding adaptive evolution of genome structure, genes, and population diversity among members of the C. gloeosporioides species complex, and also help in understanding the mechanisms of host-pathogen interactions and improve management strategies of anthracnose.
\end{abstract}

Persimmon (Diospyros kaki Thunb.), which presumably originated in China, is known as the "oriental product of beauty" due to its attractive fruit (Luo and Wang 2008). Based on Food and Agriculture Organization data (FAO 2019), the persimmon cultivated area in China in 2019 was 914,883 ha, with an estimated production of $3,207,516$ tons of edible fruit, accounting for $75 \%$ of the world total production.

Anthracnose is a devastating disease found widely in the persimmon-producing countries of the world (Carraro et al. 2019; Hassan et al. 2018; Wang et al. 2016; Williamson and Sutton 2010). It causes defoliation, new shoot blight or stem-end wither, followed by premature fruit drop and plant death, which seriously affect fruit productivity and quality (Hassan et al. 2018; Wang et al. 2016; Xie et al. 2010). Thus far, to our knowledge, nine Colletotrichum spp. (Colletotrichum acutatum, C. coccodes, C. fructicola, C. gloeosporioides sensu stricto, C. horii, C. karsti, C. melonis, C. nymphaeae, and C. siamense) have been reported to cause persimmon anthracnose (Carraro et al. 2019; Hassan et al. 2018; Jang et al. 2019; Wang et al. 2016; Williamson and Sutton 2010; Xie et al. 2010). Among them, whole or draft genome sequences of all species except C. horii, C. melonis, and C. nymphaeae are available in the GenBank (https://www.ncbi.nlm.nih.gov/genome). As the primary pathogen causing persimmon anthracnose, C. horii, initially assumed as Gloeosporium kaki (Hori 1910) and C. gloeosporioides (Von Arx 1970), was later identified as a distinct species of the $C$. gloeosporioides complex, which is composed of more than 20 closely related species, based on morphological characteristics and multilocus phylogenetic analysis (Fu et al. 2019; Weir and Johnston 2010; Weir et al. 2012). Currently, C. horii has been reported in different countries (Hassan et al. 2018; Weir and Johnston 2010; Xie et al. 2010) but little is known about the species at the genomic level. In this study, the genome of $C$. horii strain SD010 was sequenced, providing insight into the pathogenic mechanism and serving as a valuable resource for comparative genomics of fungal pathogens of persimmon anthracnose.

C. horii SD010 was isolated from the infected twigs (with necrotic lesions) of persimmon in Qingzhou, Shandong Province, China (36 68 N; $118^{\circ} 47$ E) in 2017. The identification and pathogenicity of SD010 was confirmed by both the morphological and phylogenetic analyses of five loci (internal transcribed spacer, glyceraldehyde 3-phosphate dehydrogenase, actin,

\footnotetext{
†Corresponding authors: C. X. Ai; chengxiang_1975@163.com, and R. Gao; gaorui368@163.com
}

The author(s) declare no conflict of interest.

\section{Funding}

This research was supported by the National Natural Science Foundation of China (31701899) and the Key R\&D Program of Shandong Province (2018GNC110013).

\section{Keywords}

Colletotrichum, Diospyros kaki, disease management, forest, fruit, fungi, tree fruits 
Table 1. Complete genome summary of Colletotrichum horii strain SD010

\begin{tabular}{ll} 
Variables & Statistics \\
Assembly size (bp) & $75,154,806$ \\
Number of contigs & 53 \\
GC content (\%) & 46.44 \\
Contig N $_{50}(\mathrm{bp})$ & $2,568,590$ \\
Contig N90 $_{90}(\mathrm{bp})$ & 594,756 \\
Longest sequence length (bp) & $7,214,349$ \\
BUSCO completeness & $98.1 \%$ \\
Number of predicted genes & 15,112 \\
Average gene length (bp) & 1651.77 \\
Average protein length (aa) & 463.09 \\
\hline
\end{tabular}

${ }^{a}$ Benchmarking universal single-copy orthologs.

$\beta$-tubulin 2, and chalcone synthase 1), and an inoculation test on healthy persimmon branches and reisolation test, respectively (unpublished). Total DNA was extracted by using an E.Z.N.A. High-Performance Fungal DNA Kit (Omega Bio-tek Inc., Guangzhou, China) from fungal mycelia collected from single-spore cultures. The genome of $C$. horii SD010 was sequenced using both PacBio Sequel II System (Pacific Biosciences [PacBio], Menlo Park, CA, U.S.A.) and Illumina HiSeq 4000 system (paired-end sequencing; $2 \times 150$ bp) (Illumina, San Diego, CA, U.S.A.). Genomic DNA was sheared into fragments (15 kb) using a g-TUBE (Covaris M220 Focused-ultrasonicator; Thermo Fisher Scientific, Inc. Woburn, MA, U.S.A.) and a SMRTbell Express Template Prep Kit 2.0 (PacBio) was used to construct the PacBio Sequel II library, while the Illumina TruSeq Nano DNA Sample Prep Kit (300 bp insert size) was used to generate a library for Illumina sequencing.

PacBio sequencing generated a total of 1,426,915 filtered subreads, with an average length of $8.37 \mathrm{~kb}$, an $\mathrm{N}_{50}$ of $9.72 \mathrm{~kb}$, and coverage of $160 \times$. Illumina sequencing generated approximately $18,600,018$ clean reads $(99.84 \%$ of the total reads), with $40 \times$ coverage. PacBio subreads were assembled using FALCON v0.3.0 (Chin et al. 2016) into contigs. The contigs were further corrected by the Illumina short reads using Pilon v1.16 (Walker et al. 2014). Finally, a 75,154,806-bp assembled genome containing 53 scaffolds was obtained, with a GC content of $46.4 \%, N_{50}$ of $2,568,590 \mathrm{bp}, L_{50}$ of 8 , and the largest scaffold size of $7,214,349 \mathrm{bp}$ (Table 1). Among the published genomes of the C. gloeosporioides species complex, the present genome is the largest one compared with the C. gloeosporioides, C. fructicola, C. siamense, and C. asianum genomes (Alkan et al. 2013; Gan et al. 2013; Liang et al. 2018; Meng et al. 2019, 2020). Furthermore, the completeness of the genome assembly was evaluated using benchmarking universal single-copy orthologs (BUSCO) v3.0.2 based on the lineagespecific profile library sordariomyceta_odb9 (Simão et al. 2015). The analysis revealed that the assembly had a BUSCO completeness of $98.1 \%$ (97.9\% complete and single-copy BUSCOs, $0.2 \%$ complete and duplicated BUSCOs) and $0.8 \%$ missing BUSCOs from a total of 3,725 BUSCO groups.

Analysis of repeat sequences was performed by using Repeat Masker v4.0.5 (http:// www.repeatmasker.org) based on the filtered custom RepeatModeler library generated by de novo-based method, and de novo repeats detection was performed using the Repeat Modeler v1.0.7 pipeline with default settings (Saha et al. 2008). Approximately $21.42 \%$ of sequences were identified as repeat sequences. Then, the protein-coding genes were predicted using the homology-based prediction program Exonerate v2.4.7 (Slater and Birney 2005) and two ab initio gene annotation programs, Augustus v3.3.3 (Stanke et al. 2008) and GeneMark-ES v4.38 (Lomsadze et al. 2005). A self-training algorithm was used to predict genes by GeneMark-ES and the gene models of the $C$. horii SD010 predicted by Exonerate and GeneMark-ES were used to train Augustus. The predicted results were further integrated using EVidenceModeler v1.1.1 (Haas et al. 2008), and 15,112 protein-coding genes were identified. Among these, 2,101 secretory protein-coding genes, 26 laccases, 1,131 peptidases, and 518 transcription factors were identified with the databases FunsecKB2, LccED v6.4, MEROPS v12.1, and FTFD v1.2, respectively, by BLASTP (Meinken et al. 2014; Park et al. 2008a; Rawlings et al. 2014; Sirim et al. 2011). Among the secretomes, 536 proteins (25.5\%, less than 300 amino acids) were small secreted proteins, which were considered as candidate secreted effectors (Kim et al. 2016). Other previously reported candidate virulence 
genes, including putative cytochrome P450s, carbohydrate active enzymes (CAZymes), and secondary metabolite synthetases, were also identified from the genome of SD010 in this study (Huang et al. 2019; Kleemann et al. 2012; Liang et al. 2018; Meng et al. 2020). In all, 342 fungal cytochrome P450s and 986 CAZymes were identified using FCPD v1.2, and dbCAN v6.0 databases, with an e-value < 1e-15 (Park et al. 2008b; Yin et al. 2012). In total, 61 secondary metabolite clusters (23 polyketide synthase clusters, 20 nonribosomal peptidesynthetase clusters, 10 terpene clusters, 6 indole clusters, $1 \beta$-lactone cluster, and 1 fungalPiPP cluster) were identified in the $C$. horii SD010 genome using antiSMASH v4.0 (Blin et al. 2017). Furthermore, a whole-genome comparison with other species of the C. gloeosporioides complex using MUMmer v3.23 revealed a similar association of $C$. horii with $C$. gloeosporioides Cg-14 (77.7\%), C. fructicola Nara gc5 (77.1\%), and C. fructicola 1104-7 (75.7\%) (Alkan et al. 2013; Gan et al. 2013; Liang et al. 2018). The ortholog clustering analysis using OrthoFinder v2.2.6 (Emms and Kelly 2015) showed 3,377 orthogroups specific to the species complex, including 1,168 shared among the closed four strains and 60 shared between C. horii SD010 and C. gloeosporioides Cg-14 (67 genes in SD010 and 65 genes in Cg-14). Meanwhile, 617 lineage-specific genes were identified in SD010 (unclustered or clustered only with proteins from the same genome). Among them, 549 were orphan genes, and the top three enriched Pfam domains were related to oxidoreductase (26 genes), cation binding (19 genes), and mycotoxin metabolic process (3 genes).

To conclude, this is the first report of a whole-genome sequence of $C$. horii, which provide a reference for the classification and population diversity analysis of the $C$. gloeosporioides species complex. The sequenced genome will also help in understanding the host-pathogen interaction. The genome assembly of $C$. horii SD010 was deposited at GenBank with the accession number JAGTUO000000000 (BioProject: PRJNA725041; BioSample: SAMN18868286). The version described in this article is version JAGTU0010000000. C. horii SD010 has been deposited in the China Forestry Culture Collection Center with the preservation number CFCC 55691.

\section{Acknowledgments}

We thank TopEdit (https://topeditsci.com/) for linguistic assistance during preparation of this manuscript.

\section{Literature Cited}

Alkan, N., Meng, X. C., Friedlander, G., Reuveni, E., Sukno, S., Sherman, A., Thon, M., Fluhr, R., and Prusky, D. 2013. Global aspects of pacC regulation of pathogenicity genes in Colletotrichum gloeosporioides as revealed by transcriptome analysis. Mol. Plant-Microbe Interact. 26:1345-1358.

Blin, K., Wolf, T., Chevrette, M. G., Lu, X., Schwalen, C. J., Kautsar, S. A., Suarez Duran, H. G., de Los Santos, E., Kim, H. U., Nave, M., Dickschat, J. S., Mitchell, D. A., Shelest, E., Breitling, R., Takano, E., Lee, S. Y., Weber, T., and Medema, M. H. 2017. antiSMASH 4.0-Improvements in chemistry prediction and gene cluster boundary identification. Nucleic Acids Res. 45:W36-W41.

Carraro, T. A., Lichtemberg, P. S. F., Michailides, T. J., Pereira, W. V., Figueiredo, J. A. G., and May-De Mio, L. L. 2019. First report of Colletotrichum fructicola, C. nymphaeae, and $C$. melonis causing persimmon anthracnose in Brazil. Plant Dis. 103:2692.

Chin, C. S., Peluso, P., Sedlazeck, F. J., Nattestad, M., Concepcion, G. T., Clum, A., Dunn, C., O'Malley, R., Figueroa-Balderas, R., Morales-Cruz, A., Cramer, G. R., Delledonne, M., Luo, C., Ecker, J. R., Cantu, D., Rank, D. R., and Schatz, M. C. 2016. Phased diploid genome assembly with single-molecule real-time sequencing. Nat. Methods 13:1050-1054.

Emms, D. M., and Kelly, S. 2015. OrthoFinder: Solving fundamental biases in whole genome comparisons dramatically improves orthogroup inference accuracy. Genome Biol. 16:157.

FAO. 2019. FAOSTAT Food and Agriculture Data. Food and Agriculture Organization of the United Nations. https://www.fao.org/faostat/en/\#home

Fu, M., Crous, P. W., Bai, Q., Zhang, P. F., Xiang, J., Guo, Y. S., Zhao, F. F., Yang, M. M., Hong, N., Xu, W. X., and Wang, G. P. 2019. Colletotrichum species associated with anthracnose of Pyrus spp. in China. Persoonia 42:1-35.

Gan, P., Ikeda, K., Irieda, H., Narusaka, M., O'Connell, R. J., Narusaka, Y., Takano, Y., Kubo, Y., and Shirasu, K. 2013. Comparative genomic and transcriptomic analyses reveal the hemibiotrophic stage shift of Colletotrichum fungi. New Phytol. 197:1236-1249.

Haas, B. J., Salzberg, S. L., Zhu, W., Pertea, M., Allen, J. E., Orvis, J., White, O., Buell, C. R., and Wortman, J. R. 2008. Automated eukaryotic gene structure annotation using EVidenceModeler and the program to assemble spliced alignments. Genome Biol. 9:R7.

Hassan, O., Jeon, J. Y., Chang, T., Shin, J. S., Oh, N. K., and Lee, Y. S. 2018. Molecular and morphological characterization of Colletotrichum species in the Colletotrichum gloeosporioides complex associated with persimmon anthracnose in South Korea. Plant Dis. 102:1015-1024.

Hori, S. 1910. Kaki no Shinbyogai Tansobyo. Engei no Tomo 6:58-61.

Huang, L., Kim, K. T., Yang, J. Y., Song, H., Choi, G., Jeon, J., Cheong, K., Ko, J., Xu, H., and Lee, Y. H. 2019. A high-quality draft genome sequence of Colletotrichum gloeosporioides sensu stricto SMCG1\#C, a causal agent of anthracnose on Caunninghamia lanceolata in China. Mol. Plant-Microbe Interact. 32:139-141.

Jang, S. J., Kim, S. S., and Kuk, Y. I. 2019. Effect of plant extracts and emulsifiers on control of anthracnose (Colletotrichum coccodes) in persimmon trees. Biol. Agric. Hortic. 35:123-131.

Kim, K. T., Jeon, J., Choi, J., Cheong, K., Song, H., Choi, G., Kang, S., and Lee, Y. H. 2016. Kingdom-wide analysis of fungal small secreted proteins (SSPs) reveals their potential role in host association. Front. Plant Sci. 7:186.

Kleemann, J., Rincon-Rivera, L. J., Takahara, H., Neumann, U., Ver Loren van Themaat, E., van der Does, H. C., Hacquard, S., Stüber, K., Will, I., Schmalenbach, W., Schmelzer, E., and O'Connell, R. J. 2012. Sequential delivery of host-induced virulence effectors by appressoria and intracellular hyphae of the phytopathogen Colletotrichum higginsianum. PLoS Pathog 8:e1002643.

Liang, X., Shang, S., Dong, Q., Wang, B., Zhang, R., Gleason, M. L., and Sun, G. 2018. Transcriptomic analysis reveals candidate genes regulating development and host interactions of Colletotrichum fructicola. BMC Genomics 19:557. 
Lomsadze, A., Ter-Hovhannisyan, V., Chernoff, Y. O., and Borodovsky, M. 2005. Gene identification in novel eukaryotic genomes by self-training algorithm. Nucleic Acids Res. 33:6494-6506.

Luo, Z., and Wang, R. 2008. Persimmon in China: Domestication and traditional utilizations of genetic resources. Adv. Hortic. Sci. 22:239-243.

Meinken, J., Asch, D. K., Neizer-Ashun, K. A., Chang, G.-H., Cooper, C. R., Jr., and Min, X. J. 2014. FunSecKB2: A fungal protein subcellular location knowledgebase. Comput. Mol. Biol. 4:1-17.

Meng, Y., Ren, Y., Wang, W., Gleason, M. L., Zhang, R., and Sun, G. 2020. A genome sequence resource for the geographically widespread anthracnose pathogen Colletotrichum asianum. Plant Dis. 104:2044-2047.

Meng, Y. N., Gleason, M. L., Zhang, R., and Sun, G. Y. 2019. Genome sequence resource of the wide-host-range anthracnose pathogen Colletotrichum siamense. Mol. Plant-Microbe Interact. 32:931-934.

Park, J., Jang, S., Kim, S., Kong, S., Choi, J., Ahn, K., Kim, J., Lee, S., Kim, S., Park, B., Jung, K., Kim, S., Kang, S., and Lee, Y. H. 2008a. FTFD: An informatics pipeline supporting phylogenomic analysis of fungal transcription factors. Bioinformatics 24:1024-1025.

Park, J., Lee, S., Choi, J., Ahn, K., Park, B., Park, J., Kang, S., and Lee, Y. H. 2008b. Fungal cytochrome P450 database. BMC Genomics 9:402.

Rawlings, N. D., Waller, M., Barrett, A. J., and Bateman, A. 2014. MEROPS: The database of proteolytic enzymes, their substrates and inhibitors. Nucleic Acids Res. 42:D503-D509.

Saha, S., Bridges, S., Magbanua, Z. V., and Peterson, D. G. 2008. Empirical comparison of ab initio repeat finding programs. Nucleic Acids Res. 36:2284-2294.

Simão, F. A., Waterhouse, R. M., loannidis, P., Kriventseva, E. V., and Zdobnov, E. M. 2015. BUSCO: Assessing genome assembly and annotation completeness with single-copy orthologs. Bioinformatics 31:3210-3212.
Sirim, D., Wagner, F., Wang, L., Schmid, R. D., and Pleiss, J. 2011. The Laccase Engineering Database: A classification and analysis system for laccases and related multicopper oxidases. Database 2011:bar006.

Slater, G. S., and Birney, E. 2005. Automated generation of heuristics for biological sequence comparison. BMC Bioinformatics 6:31.

Stanke, M., Diekhans, M., Baertsch, R., and Haussler, D. 2008. Using native and syntenically mapped cDNA alignments to improve de novo gene finding. Bioinformatics 24:637-644.

Von Arx, J. A. 1970. A revision of fungi classified as Gloeosporium. Bibl. Mycol. 24:1203.

Walker, B. J., Abeel, T., Shea, T., Priest, M., Abouelliel, A., Sakthikumar, S., Cuomo, C. A., Zeng, Q., Wortman, J., Young, S. K., and Earl, A. M. 2014. Pilon: An integrated tool for comprehensive microbial variant detection and genome assembly improvement. PLoS One 9:e112963.

Wang, J., Ai, C. X., Yu, X. M., An, M., Sun, S., and Gao, R. 2016. First report of Colletotrichum karstii causing anthracnose on persimmon leaves in China. Plant Dis. 100:532.

Weir, B. S., and Johnston, P. R. 2010. Characterisation and neotypification of Gloeosporium kaki Hori as Colletotrichum horii nom. nov. Mycotaxon 111:209-219.

Weir, B. S., Johnston, P. R., and Damm, U. 2012. The Colletotrichum gloeosporioides species complex. Stud. Mycol. 73:115-180.

Williamson, S. M., and Sutton, T. B. 2010. First report of anthracnose caused by Colletotrichum acutatum on persimmon fruit in the United States. Plant Dis. 94:634.

Xie, L., Zhang, J. Z., Cai, L., and Hyde, K. D. 2010. The biology of Colletotrichum horii, the causal agent of Persimmon anthracnose. Mycology 1:242-253.

Yin, Y., Mao, X., Yang, J., Chen, X., Mao, F., and Xu, Y. 2012. dbCAN: A web resource for automated carbohydrate-active enzyme annotation. Nucleic Acids Res. 40:W445-W451. 